\title{
Review and Analysis of Two Gitelman Syndrome Pedigrees Complicated with Proteinuria or Hashimoto's Thyroiditis Caused by Compound Heterozygous SLC12A3 Mutations
}

\author{
Jian-hui Zhang $\mathbb{D}^{1,2}$ Dan-dan Ruan $\mathbb{D}^{1,2}$ Ya-nan Hu ${ }^{10},{ }^{1,2}$ Xing-lin Ruan $\mathbb{D}^{3},{ }^{3}$ Yao-bin Zhu $\mathbb{D}^{4}$,

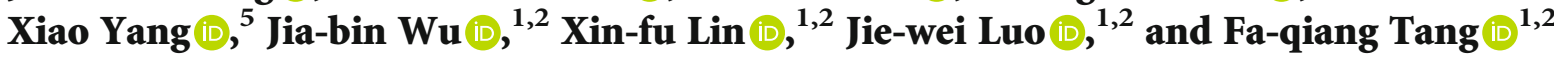 \\ ${ }^{1}$ Shengli Clinical Medical College of Fujian Medical University, Fuzhou 350001, China \\ ${ }^{2}$ Fujian Provincial Hospital, Fuzhou 350001, China \\ ${ }^{3}$ Department of Neurology, Fujian Medical University Union Hospital, Fuzhou 350001, China \\ ${ }^{4}$ Department of Traditional Chinese Medicine the First Affiliated Hospital, Fujian Medical University, Fuzhou 350001, China \\ ${ }^{5}$ Teaching and Research Office of Medical Cosmetology, Department of Management, Fujian Health College, Fuzhou 350001, China
}

Correspondence should be addressed to Jie-wei Luo; docluo0421@aliyun.com and Fa-qiang Tang; faqiangtang@fjmu.edu.cn

Received 18 March 2021; Revised 25 April 2021; Accepted 3 May 2021; Published 10 May 2021

Academic Editor: Maria Irene Bellini

Copyright ( 2021 Jian-hui Zhang et al. This is an open access article distributed under the Creative Commons Attribution License, which permits unrestricted use, distribution, and reproduction in any medium, provided the original work is properly cited.

Gitelman syndrome (GS) is an autosomal recessive inherited salt-losing renal tubular disease, which is caused by a pathogenic mutation of SLC12A3 encoding thiazide-sensitive $\mathrm{Na}-\mathrm{Cl}$ cotransporter, which leads to disturbance of sodium and chlorine reabsorption in renal distal convoluted tubules, resulting in phenotypes such as hypovolemia, renin angiotensin aldosterone system (RAAS) activation, hypokalemia, and metabolic alkalosis. In this study, two GS families with proteinuria or Hashimoto's thyroiditis were analyzed for genetic-phenotypic association. Sanger sequencing revealed that two probands carried SLC12A3 compound heterozygous mutations, and proband A carried two pathogenic mutations: missense mutation Arg83Gln, splicing mutation, or frameshift mutation NC_000016.10:g.56872655_56872667 (gcggacatttttg>accgaaaatttt) in exon 8. Proband B carries two missense mutations: novel Asp839Val and Arg904Gln. Both probands manifested hypokalemia, hypomagnesemia, hypocalcinuria, metabolic alkalosis, and RAAS activation; in addition, the proband A exhibited decreased urinary chloride, phosphorus, and increased magnesium ions excretion, complicated with Hashimoto's Thyroiditis, while the proband B exhibited enhanced urine sodium excretion and proteinuria. The older sister of proband B with GS also had Hashimoto's thyroiditis. Electron microscopy revealed swelling and vacuolar degeneration of glomerular epithelial cells, diffuse proliferation of mesangial cells and matrix, accompanied by a small amount of low-density electron-dense deposition, and segmental fusion of epithelial cell foot processes in proband B. Light microscopy showed mild mesangial hyperplasia in the focal segment of the glomerulus, hyperplasia, and hypertrophy of juxtaglomerular apparatus cells, mild renal tubulointerstitial lesions, and one glomerular sclerosis. So, long-term hypokalemia of GS can cause kidney damage and may also be susceptible to thyroid disease.

\section{Introduction}

Gitelman syndrome (GS) is an autosomal recessive hereditary salt-losing renal tubular disease. Hillel J. Gitelman reported three familial diseases characterized by hypokalemia, hypomagnesemia, hypochloremic alkalosis, and hypocalciuria in 1966, and the syndrome was named after him [1]. The prevalence of GS is approximately 1-10 per 40000 people, and the prevalence of heterozygotes is about $1 \%$ in western countries [2, 3]. However, in Asia, the prevalence of GS has significantly increased to 10.3 per 10000 people [4], and mutations may be as high as 3\% [3]. China reports are fewer, and the number of samples in these reports is comparatively small. GS is inherited in an autosomal recessive mode, and its pathogenic gene is the SLC12A3 gene (MIM:600968; NC_000016.10), which is located on human chromosome 16q13 and consists of 26 independent exons [5]. Currently, a variety of pathogenic mutations have been 
discovered, including nonsense mutations, missense mutations, frameshift mutations, deletions, insertions, and splice site mutations [6]. The hot spot mutation site is not yet known. In this study, two GS pedigrees with compound heterozygous mutation presenting with proteinuria or Hashimoto's thyroiditis phenotype were reported. We observed damage to renal tissue in the case of SLC12A3 mutations causing GS through renal biopsy and discussed the possible relationship between GS and renal pathology and thyroid function.

\section{Materials and Methods}

2.1. Subjects. The study included 16 cases from 2 GS pedigrees, specifically 3 males and 6 females from Pedigree A and 5 males and 2 females from Pedigree $B$. Both pedigrees had no consanguineous marriages. Proband II6 (proband A) of Pedigree A, a female, aged 42, married, with a blood pressure of $124 / 76 \mathrm{mmHg}$, and a BMI of $21.22 \mathrm{~kg} / \mathrm{m}^{2}$, was admitted to the hospital with the chief complaint of "repeated fatigue for one year and chest tightness for one month." The serum potassium checked locally was $2.95 \mathrm{mmol} / \mathrm{L}$, the chest tightness and palpitations occurred one month before admission, and general fatigue was obvious. The mother of proband A had long-term hypokalemia with no relevant clinical manifestations. Proband II3 (proband B) of Pedigree B, a male, aged 31 ,with a blood pressure of $125 / 79 \mathrm{mmHg}$, and a BMI of $21.52 \mathrm{~kg} / \mathrm{m}^{2}$, was admitted to the hospital because of "repeated fatigue and foamy urine for half a year." The proband had increased urine foam with no obvious induction half a year before admission, no decrease in urine output, and no swelling on the face or lower limbs. After testing at the local hospital, the serum potassium level was determined to be $2.33 \mathrm{mmol} / \mathrm{L}$, and this level fluctuated from 2.3 to $2.7 \mathrm{mmol} / \mathrm{L}$ after oral potassium supplementation. The older sister of proband B had chronic hypokalemia, which mainly manifested as paroxysmal weakness of limbs and muscle spasms. None of the patients had a low potassium diet or complained of long-term vomiting, diarrhea, or other symptoms. They also had no history of overuse of diuretics, laxatives, or alcohol and no history of drug addiction. No other extrarenal and renal causes of hypokalemia, such as Cushing syndrome, primary aldosteronism, reninoma, Liddle syndrome, renal tubular acidosis, diabetic ketoacidosis, and renal artery stenosis, were found, and there was no history of nephrotoxic drugs or licorice intake. This study was approved by the Ethics Committee of Fujian Provincial Hospital, and all family members participating in this study provided signed informed consent.

2.2. Detection Methods. Genomic DNA was extracted from peripheral blood. Under the principle of informed consent, peripheral blood samples $(2 \mathrm{~mL})$ were extracted from the patient, and DNA was extracted using the centrifugal column method. The DNA extraction kit used was the QIAGEN DNA whole blood extraction kit of the Gene Company (QIAGEN DNA whole blood extraction kit; QIAGEN, Hilden, Germany).

For primer design, the $S L C 12 A 3$ gene sequence was obtained from GenBank (NC_000016) (MIM:600968), and 26 pairs of primers were designed using primer premier 5 soft- ware to amplify all 26 exons of the SLC12A3 gene (as shown in Table 1). The primers were synthesized by the Beijing Liuhe Huada GenePolytron Technologies Inc.

For PCR product amplification, the reaction system $(30 \mu \mathrm{L})$ contained $2.5 \mu \mathrm{L} 10 \times$ Ex Taq buffer, $2 \mu \mathrm{L}$ dNTP (2.5 mM), $3 \mu \mathrm{L}$ forward primer $(3.0 \mathrm{mM}), 3 \mu \mathrm{L}$ reverse primer (3.0 mM), $1 \mu \mathrm{L}$ DNA template $1,0.2 \mu \mathrm{L} \mathrm{Ex} \mathrm{Taq,} \mathrm{and} 18.3 \mu \mathrm{L}$ $\mathrm{H}_{2} \mathrm{O}$. Using the PCR instrument (PTC-200 PCR, BIO$\mathrm{RAD}$ ), denaturing was started at $94^{\circ} \mathrm{C}$ for $5 \mathrm{~min}$, followed by denaturation at $94^{\circ} \mathrm{C}$ for $40 \mathrm{~s}$ (each primer TM value), annealing for $40 \mathrm{~s}$, extension at $72^{\circ} \mathrm{C}$ for $60 \mathrm{~s}, 35$ times circulation, and then extension at $72^{\circ} \mathrm{C}$ for $10 \mathrm{~min}$.

The PCR products were purified using the methods described in the operating instructions of $\mathrm{EZNA}^{\mathrm{TM}} \mathrm{Gel}$ Extraction Kit (Omega Company), and the sequencing was performed according to the PCR product standard operating procedure of BigDye Terminator v1.1 kit.

For analysis of the DNA sequencing results, the DNAMAN Version 5.2.2 was used for alignment with normal sequences. When a heterozygous deletion or insertion was suspected, PCR products were connected to the PGEM-T Easy (Promega) vector to select subclones for sequencing [7].

\section{Results}

3.1. Clinical Phenotype. Among the 16 cases in the two GS pedigrees, 4 cases, the proband of Pedigree A (II6), her older sister (II5), the proband of Pedigree B (II3), and his older sister (II2), met the clinical diagnostic criteria of GS: chronic persistent hypokalemia ( $<3.5 \mathrm{mmol} / \mathrm{L})$, hypomagnesemia $(<0.7 \mathrm{mmol} / \mathrm{L})$, and hypocalcinuria (urinary calcium/urinary creatinine < $0.2 \mathrm{mmol} / \mathrm{mmol}$ ) [8]. However, the mother (I2) of proband A of Pedigree A had long-term mild hypokalemia (serum potassium ranges from 3.0 to $3.5 \mathrm{mmol} / \mathrm{L}$ ), but comprehensive biochemical indicators had not been collected, and there was insufficient evidence for GS diagnosis. Both probands showed hypokalemia, hypomagnesemia, hypocalcinuria, metabolic alkalosis, blood renin, and angiotensin activation state, and proband A showed decreased urine chloride and phosphorus with increased magnesium ion excretion and thyroid dysfunction. The thyroid color doppler ultrasound of Proband A indicated mild diffuse enlargement of the thyroid gland with clear boundaries and no space occupation; however, the thyroid peroxidase and TG antibodies were elevated, resulting in a diagnosis of "Hashimoto's thyroiditis." In addition, the electronic gastroscope showed chronic superficial gastritis, and proton pump inhibitors were administered for treatment. Proband $\mathrm{B}$ had increased urine sodium excretion, a positive urine routine protein $(++)$, and a significantly excessive $24 \mathrm{~h}$ urine protein level. Both patients had normal blood pressure, weakness and numbness of limbs, and occasional heart palpitations, chest tightness, and discomfort. Proband B also showed proteinuria. The older sister of proband B had chronic hypokalemia, accompanied by thyroid dysfunction and elevated thyroid peroxidase antibodies and TG antibodies. The thyroid color doppler ultrasound indicated mild and diffuse thyroid enlargement, which was similar to the result of proband A, and this also led to a diagnosis of "Hashimoto's thyroiditis" (Figure 1, Table 2). 
TABLE 1: The exon primer and PCR condition of SLC12A3 gene.

\begin{tabular}{|c|c|c|c|c|}
\hline \multirow{2}{*}{ Exon } & \multicolumn{2}{|c|}{ Primer sequence $\left(5^{\prime}-3^{\prime}\right)$} & \multirow{2}{*}{$\begin{array}{l}\text { Annealing temperature } \\
\qquad\left({ }^{\circ} \mathrm{C}\right)\end{array}$} & \multirow{2}{*}{$\begin{array}{l}\text { Length } \\
\text { (bp) }\end{array}$} \\
\hline & Forward & Reverse & & \\
\hline 1 & CTATAAAACCACCCTGTGTGTCCTT & GAAGTGGCCAGTCTTCTGAGAC & 55 & 374 \\
\hline 2 & ССТCAAGCAGCTCAACACC & GCCTGAGAGTCAGAGCTGAG & 55 & 375 \\
\hline 3 & TACTGAAGTGGGTGAAGAAGGGA & CTGAGACTGAACCAGGGAGGAGAA & 60 & 372 \\
\hline 4 & CTCCCAAAGTGACAGAGACCCAT & GGGAGTCCTGTTCCCAGGATTA & 55 & 311 \\
\hline 5 & CCAACCGACTCATCTGGTTTCA & САСТСТСАСССАCAGTGATCAG & 55 & 330 \\
\hline 6 & AACATCGTCCTAGCAGAGTGC & TCCAGCATGGACATCGAGC & 60 & 372 \\
\hline 7 & CTTGAACAGATCCTGCTGCATAATG & CCTGACCATGGGATTGGGTAAT & 55 & 375 \\
\hline 8 & TGGGAGGATGGGATTACCCAAT & GGACTGGACTGGATTTTAGAAGCC & 52 & 372 \\
\hline 9 & CGACCCGTGATCTTGGTTGTAT & CACTTACCAATGGTGGCTGAGAT & 60 & 329 \\
\hline 9 & GCCATTTTCTGGACGACCATTT & TCTCCCTGTTGTTAGAGAGTCGAA & 60 & 171 \\
\hline 10 & GCTGGAAGAGGACAGAGTAAGGA & СТСТССТАAGCСТAGGCСТСAA & 60 & 344 \\
\hline 11 & GTAGGGAATGAAGTGCCACAGAT & CCTTCAGGTGTTTGTAGCAGTCA & 60 & 286 \\
\hline 12 & GCAACTCCACCATTCAAGCTCT & AGCCTTACCGATGATGATGAAGG & 55 & 375 \\
\hline 12 & AGGCTATGGCAAGAACAAGGAG & CTCTTAGTGCCCACTAACTGTCAG & 55 & 208 \\
\hline 13 & TCACAGATGAGAAGGTTGAGACTGA & $\begin{array}{c}\text { TCAATGGTTTTAAATTGAGAGGTGAC } \\
\text { CTT }\end{array}$ & 60 & 368 \\
\hline 14 & GGGATGTCCTGTGGCTGTATTT & CATGATGACCACGGAGATGATAGC & 60 & 330 \\
\hline 14 & $\begin{array}{l}\text { AGACCTTCATTCCAATACTACAACAA } \\
\text { GTG }\end{array}$ & GCCACATTGGGAGGGATAAAGG & 60 & 303 \\
\hline 15 & TGCCTAGAGAAGGCCGACATTA & CCATGTCTGTTCCCTCTCTGAGT & 60 & 344 \\
\hline 16 & GGAAGGACCAGGGAGACTAGTG & ACGTGGCCACAGATCATCAG & 60 & 320 \\
\hline 16 & GGACTTTGTGGGCACCTTCA & TCTGTGGGTGGACATCACAC & 60 & 230 \\
\hline 17 & CCСАCTCCTTGTGTTTTCССTTA & GACTTTCTGCCTTCCAGGTTGT & 60 & 363 \\
\hline 18 & TTTTTGAGAATCAGCACATCTGGAGA & GCCCAGCAGGACTCAACTTTTTA & 55 & 326 \\
\hline 19 & CCAATTCTGCCTGTACAGGATACA & GGGACCATTAAGAGGCGACTTT & 55 & 375 \\
\hline 20 & GGGACTTTCTTCCTAGCATTAAGGG & CACCTGTCCTCGACCAAGTT & 60 & 266 \\
\hline 21 & GAATGGAGAGTGCACTTCCСТA & GTCACTGACCTCCATCGTCAAA & 60 & 372 \\
\hline 21 & CAGGGCAAGAAGACCATAGACATC & $\begin{array}{c}\text { CTCTCAAAGCTTCCCATTTTATAACC } \\
\text { AAAA }\end{array}$ & 60 & 239 \\
\hline 22 & GCGACTTGAATTCAGTCAGCCAT & GTGGTGGTAGAGGATCTAGGGTA & 60 & 369 \\
\hline 23 & $\begin{array}{c}\text { GGTGCTCAGTGAAAATTAGTTGAATG } \\
\text { AAT }\end{array}$ & CGGAACTTGCTCAGCAGAGAA & 60 & 375 \\
\hline 23 & TCCATGTGTCCTCCAGGATCAT & СТCСТAGTCTACCAAGGAAAAAGGG & 60 & 198 \\
\hline 24 & GGGACACAATCTGATTTGTTCACTG & TCATCCTTGAAGCCATCATTCAGAC & 55 & 375 \\
\hline 24 & CACCAAGAGGTTTGAGGACATGAT & CAAGGATAGCACTGAGTTCCACA & 55 & 329 \\
\hline 25 & CTTCCTGGAGACAGGAGACTCTAT & CCAGGGCTATGTTTATGGGAACT & 60 & 374 \\
\hline 26 & GCTCTGAGGGACGGTAAACAGA & GCCACTTAAAGTGCAACAGAACAT & 55 & 362 \\
\hline
\end{tabular}

Pathological electron microscopic examination of a renal biopsy of proband B showed that the glomerular capillary endothelial cells had obvious vacuolar degeneration, evidence of red blood cells in some lumens, no obvious endothelial cell proliferation, and open capillary loops. There was no obvious thickening in the parietal layer of the renal capsule, and the parietal cells showed vacuolar degeneration with no obvious proliferation. There was swelling and vacuolar degeneration in the glomerular visceral epithelial cells, and segmental fusion of epithelial cells foot processes. The glomerular mesangial cells and matrix proliferated, accompanied by a small amount of low-density electron-dense deposition. There showed vacuolar degeneration of the renal tubular epithelial cells with no special changes in the renal interstitium. The basement membrane was not significantly thickened, as the thickness was about 300-400 nm. Light microscopy showed mild mesangial hyperplasia in the focal segment of the glomerulus, hyperplasia and hypertrophy of the juxtaglomerular apparatus, and mild renal tubulointerstitial lesions. Renal tubular epithelial cell degeneration (turbidity), no cast, interstitial edema, a small number of lymphocytes, monocytes, and foam tissue cells were observed. The immunohistochemistry results for IgG, IgM, IgA, C3d, C4d, and $\mathrm{Clq}$ were all negative (Figure 1 ). 
Pedigree A

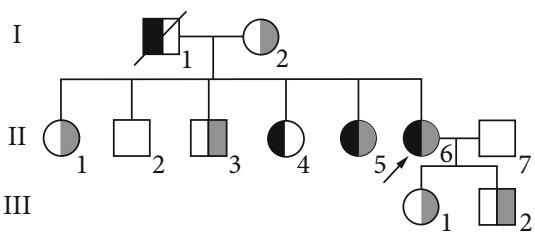

(a)

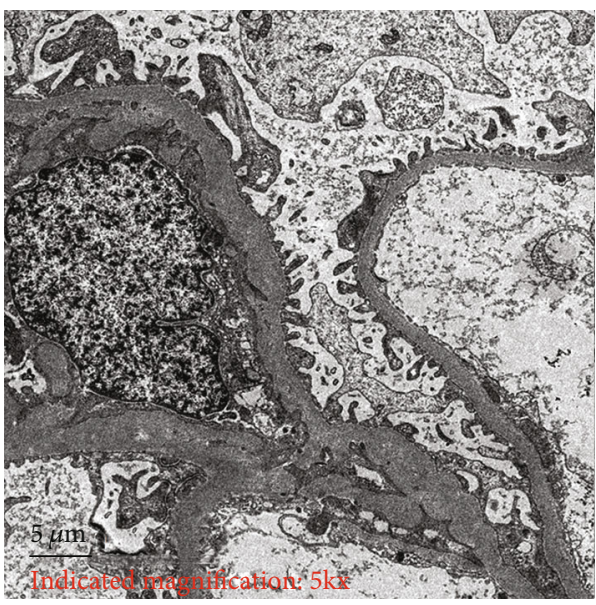

(c)

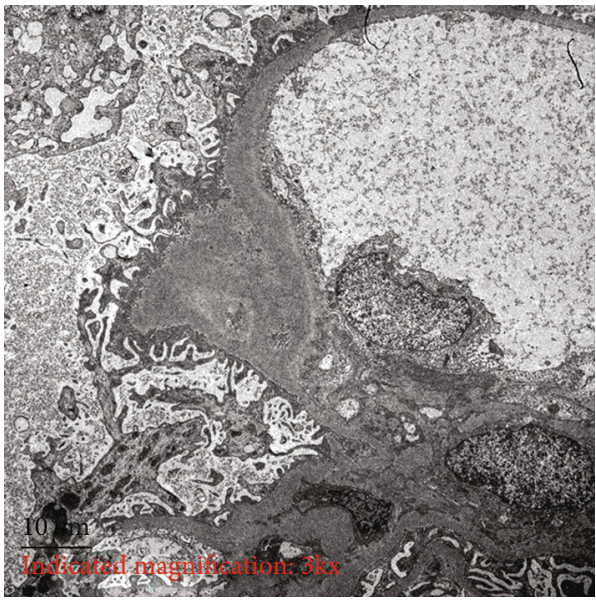

(e)

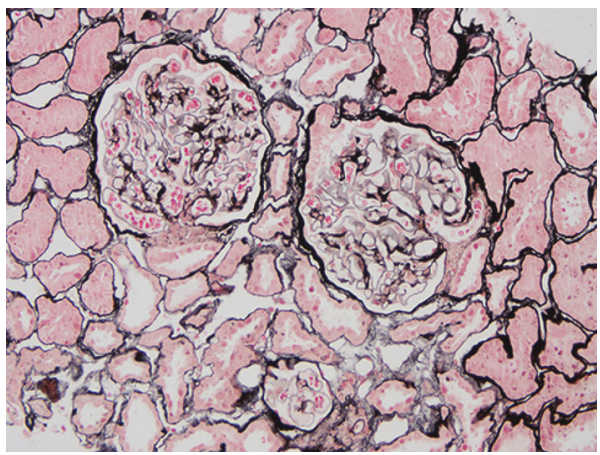

(g)

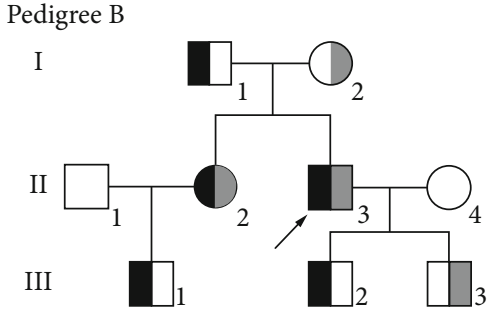

(b)

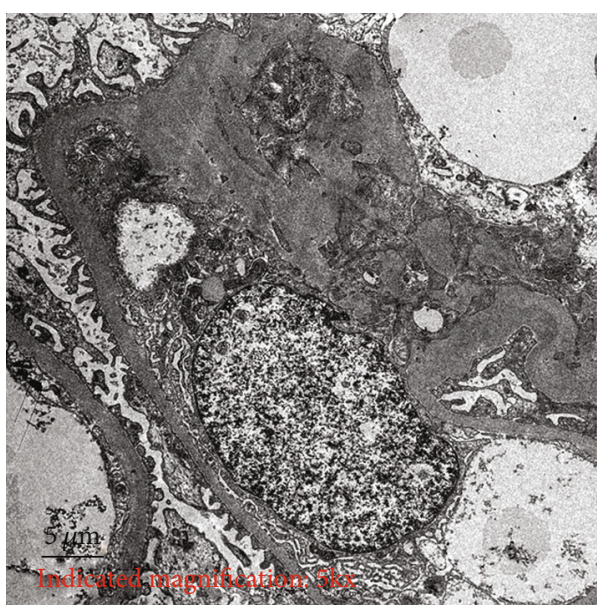

(d)

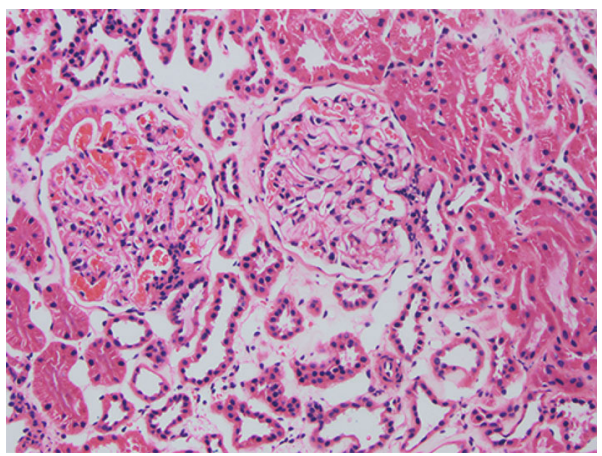

(f)

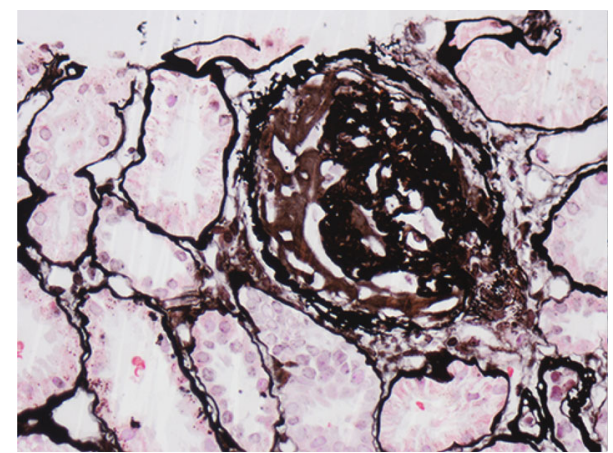

(h)

FIgURe 1: Continued. 


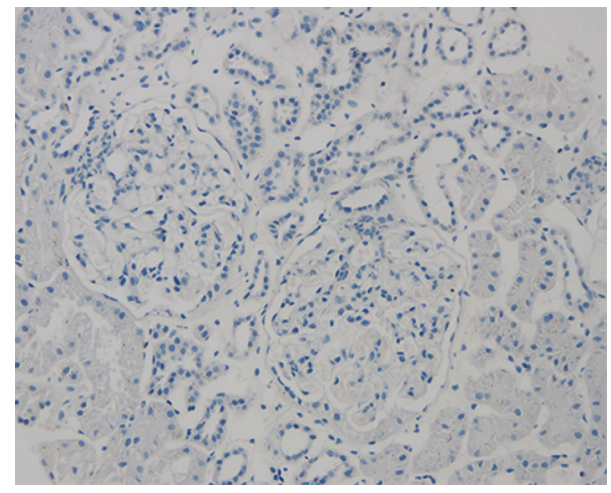

(i)

FIGURE 1: (a) Family A genetic pedigree map; black indicates a carrier of the Arg83Gln mutation, gray indicates a carrier of the mutation of SLC12A3 NC_000016.9:g.56872655_56872667 (gcggacatttttg>accgaaaatttt), the arrow indicates the proband, the square indicates male, and the circle indicates female; (b) family B genetic pedigree map; black indicates a carrier of the Asp839Val mutation, gray indicates a carrier of the Arg904Gln mutation, the arrow indicates the proband, the square indicates male, and the circle indicates female; (c-e) pathological electron microscopic examination of renal biopsy shows swelling and vacuolar degeneration of glomerular epithelial cells, diffuse proliferation of mesangial cells and matrix, accompanied by a small amount of low-density electron dense deposition, swelling, and vacuolar degeneration in the visceral epithelial cells, segmental fusion of epithelial cells foot processes. (f-i) Pathological light microscopic examination of renal biopsy shows mild mesangial hyperplasia in the focal segment of the glomerulus, hyperplasia, and hypertrophy of juxtaglomerular apparatus cells, mild renal tubulointerstitial lesions, and one glomerular sclerosis (h). (f) HE staining $\times 200$; (g, h) PAM staining $\times 200$; (i) PAS staining $\times 200$.

3.2. SLC12A3 Gene Mutation. The results of amplification and direct sequencing of SLC12A3 (NM_001126108.2) indicated that the genes of the two probands contained compound heterozygous mutations. Proband A (II6) and the older sister of proband A (II5) both had 2 suspicious pathogenic mutations, one of which was C.248G $>$ A of exon 1, which causes $\mathrm{CGG} \rightarrow \mathrm{CAG}$ changes, and leads to Arg83Gln, where arginine was substituted by glutamine resulting in a missense mutation. The other mutation point was NC_000016.10: g.56872655_ 56872667 (gcggacatttttg>accgaaaatttt) of exon 8, which is a splice site mutation or frameshift mutation; the mother of proband A (I2), as well as II1, II3, III1, and III2 only carried the mutation NC_000016.10:g.56872655_56872667 (gcggacatttttg>accgaaaatttt) heterozygotes, while II4 only carried the mutation Arg83Gln (Figure 2, Table 3). Proband B (II3) and the older sister (II2) of proband B had two mutation sites, which were: c. $2516 \mathrm{~A}>\mathrm{T}$ of exon 21 , which caused GAT $\rightarrow \mathrm{GTT}$, where aspartic acid was replaced by valine, leading to Asp839Val; and c.2711G $>A$ of exon 23 which caused $\mathrm{CGG} \rightarrow \mathrm{CAG}$, and arginine was replaced by glutamine, resulting in Arg904Gln (Figure 3). Between them, Asp839Val was inherited from their father (I1), and Arg904Gln from their mother (I2); in addition, III1 andIII2 carried Asp839Val heterozygous mutation, and III3 carried the Arg904Gln mutation. See Figure 1 and Table 3 for pedigree analysis.

\section{Discussion}

A thiazide-sensitive $\mathrm{NaCl}$ cotransporter (NCCT) is the main ion transport system of distal convoluted tubules (DCT); 5$10 \%$ of sodium and chloride ions filtered from the glomerulus are reabsorbed in DCT [9]. The SLC12A3 mutation leads to structural changes and/or dysfunctions of NCCT located in the DCT cortex; the reabsorption of $\mathrm{NaCl}$ in the DCT is reduced, and the decrease of sodium reabsorption capacity leads to an excessive exchange of sodium ions through $\mathrm{Na}+/ \mathrm{K}+$ and $\mathrm{Na}+/ \mathrm{H}+$ compensatory reabsorption. While excreting a large amount of $\mathrm{K}+$ and $\mathrm{H}+$, the reduction of reabsorption also causes hypovolemia, which promotes the synthesis and secretion of renin. This is accompanied by renal cell hyperplasia and hypertrophy, and the entire renin angiotensin aldosterone system (RAAS) is activated, which was verified by the renal biopsy pathology of proband $\mathrm{B}$ in this study. The excessive exchange of $\mathrm{Na}+/ \mathrm{K}+$ and $\mathrm{Na}+/ \mathrm{H}+$ eventually leads to hypokalemic alkalosis. At the same time, due to the large outflow of chloride ions, the polarity of the distal renal tubular cells increases, which increases the reabsorption of calcium ions, leading to hypocalciuria. A decrease in sodium ion reabsorption can lead to a related decrease in magnesium ion reabsorption, leading to hypomagnesemia. In short, these mutations can cause structural changes of NCCT that destroy its biological effects, affect its reabsorption ability, and lead to electrolyte disorders.

The clinical phenotypes of GS show great heterogeneity [10], and there is no obvious association between genotype and phenotype. A patient may show no clinical symptoms and diagnosis of hypokalemia may only occur on a routine physical examination. Electrolyte disturbances, increased RAAS activity, and other factors lead to common clinical manifestations of GS, such as weakness and numbness of limbs, paresthesia, muscle spasm, convulsion, halophilic, normal or low blood pressure, palpitation, arrhythmia, proteinuria, and hypokalemic nephropathy [11, 12]. Patients with homozygous or compound heterozygous mutations usually exhibit lower blood pressure, and approximately $2 \%$ of hypotension is caused by GS [4]. The phenomenon of hypotension was not found in these two pedigrees, which may be related to individual differences or dietary habits. 
TABLE 2: Clinical and biochemical characteristics in index cases of GS.

\begin{tabular}{|c|c|c|c|}
\hline Variable & Proband A & Proband B & Normal value \\
\hline \multicolumn{4}{|l|}{ Blood gas analysis } \\
\hline $\mathrm{PH}$ & 7.481 & 7.460 & $7.35 \sim 7.45$ \\
\hline $\mathrm{PaCO}_{2}(\mathrm{mmHg})$ & 35.2 & 40.7 & $35 \sim 45$ \\
\hline $\mathrm{BE}(\mathrm{mmol} / \mathrm{L})$ & 5.2 & 5.4 & $-3.0 \sim+3.0$ \\
\hline $\mathrm{SB}(\mathrm{mmol} / \mathrm{L})$ & 29.0 & 29.2 & $21.3 \sim 24.8$ \\
\hline $\mathrm{AB}(\mathrm{mmol} / \mathrm{L})$ & 28.0 & 29.4 & $21.4 \sim 27.3$ \\
\hline \multicolumn{4}{|l|}{ Biochemical indexes } \\
\hline Serum sodium $(\mathrm{mmol} / \mathrm{L})$ & 138 & 135 & $137 \sim 147$ \\
\hline Serum potassium $(\mathrm{mmol} / \mathrm{L})$ & 3.2 & 2.2 & $3.5 \sim 5.3$ \\
\hline Serum chloride $(\mathrm{mmol} / \mathrm{L})$ & 96 & 92 & 99 110 \\
\hline Serum calcium $(\mathrm{mmol} / \mathrm{L})$ & 2.25 & 2.34 & $2.11 \sim 2.52$ \\
\hline Serum magnesium $(\mathrm{mmol} / \mathrm{L})$ & 0.50 & 0.38 & $0.75 \sim 1.02$ \\
\hline Serum phosphate $(\mathrm{mmol} / \mathrm{L})$ & 0.87 & 0.99 & $0.85 \sim 1.51$ \\
\hline Blood urea nitrogen $(\mathrm{mmol} / \mathrm{L})$ & 3.2 & 5.37 & $2.1 \sim 7.1$ \\
\hline Creatinine $(\mathrm{mmol} / \mathrm{L})$ & 43 & 54 & $40 \sim 135$ \\
\hline $\operatorname{GFR}(\mathrm{mL} / \mathrm{min})^{\#}$ & 85.8 & 94.6 & $80 \sim 120$ \\
\hline Glucose $(\mathrm{mmol} / \mathrm{L})$ & 4.62 & 5.56 & $3.9 \sim 6.1$ \\
\hline \multicolumn{4}{|l|}{ Renin angiotensin aldosterone system } \\
\hline Renin activity $(\mu \mathrm{g} / \mathrm{L} \bullet \mathrm{h})[$ lie $]$ & 12.22 & 6.34 & $0.05 \sim 0.79$ \\
\hline Angiotensin II (ng/L) [lie] & 95.99 & 66.46 & $28.2 \sim 52.2$ \\
\hline Aldosterone (ng/L) [lie] & 116.73 & 87.47 & $59.5 \sim 173.9$ \\
\hline \multicolumn{4}{|l|}{ Corticotropin and cortisol levels } \\
\hline ACTH $(\mathrm{pg} / \mathrm{mL})[16 \mathrm{pm}]$. & 17.6 & 12.6 & $7.2 \sim 63.6$ \\
\hline Cortisol (nmol/L) [16 pm.] & 165.4 & 221.8 & $58 \sim 395$ \\
\hline \multicolumn{4}{|l|}{ Urine tests } \\
\hline Urine protein & - & ++ & - \\
\hline 24-hours proteinuria $(\mathrm{g} / 24 \mathrm{~h})$ & 0.012 & 0.823 & $<0.15$ \\
\hline 24-hour urine potassium (mmol/day) & 33.4 & 77 & $25.0 \sim 125.0$ \\
\hline 24-hour urine calcium (mmol/day) & 0.2 & 1.4 & $2.5 \sim 7.5$ \\
\hline 24-hour urine sodium (mmol/day) & 108 & 335 & $40 \sim 220$ \\
\hline 24-hour urine chlorine (mmol/day) & 115 & 223 & $170 \sim 255$ \\
\hline 24-hour urine phosphorus (mmol/day) & 6.7 & 22.2 & $12.9 \sim 42.0$ \\
\hline 24-hour urine magnesium (mmol/day) & 5.5 & 5.0 & $3.0 \sim 5.0$ \\
\hline Urine calcium/creatinine $(\mathrm{mmol} / \mathrm{mmol})$ & 0.0002 & 0.001 & $<0.2$ \\
\hline \multicolumn{4}{|l|}{ Thyroid function index ${ }^{*}$} \\
\hline TSH (mIU/L) & 6.19 & 0.94 & $0.27 \sim 4.2$ \\
\hline TPOAb (IU/mL) & $>600.00$ & 7.17 & $0 \sim 34$ \\
\hline FT3 (pmol/L) & 4.80 & 5.70 & $3.1 \sim 6.8$ \\
\hline FT4 (pmol/L) & 14.24 & 18.03 & $12 \sim 22$ \\
\hline TGAb (IU/mL) & 740.00 & $<10.00$ & $0.1 \sim 115$ \\
\hline
\end{tabular}

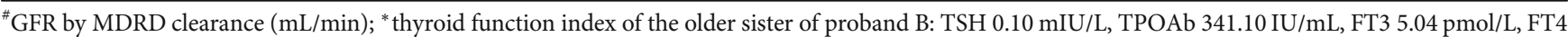
$18.93 \mathrm{pmol} / \mathrm{L}$, TGAb $545.40 \mathrm{IU} / \mathrm{mL}$ (normal value as above).

The Arg904Gln variant of SLC12A3 may increase the risk of EH (essential hypertension) [13-15], which indicates that the Arg904Gln variant may be a functional gain mutation [14]. The fatigue of GS patients is mainly caused by hypokalemia, which is generally considered to be related to the fluctuation of potassium ion concentration in or out of the cells, possibly due to excessive $\beta$-sympathetic nerve excitation or inherited mutation resulting in abnormal potassium channel activity [16]. The deficiency of potassium and magnesium prolonged the duration of the action potential of cardiomyocytes, resulting in longer QT intervals in 50\% of patients, which led to an increased risk of ventricular arrhythmia. GS patients with long-term ventricular tachycardia have been reported [17]; patients diagnosed with GS need to undergo a resting 

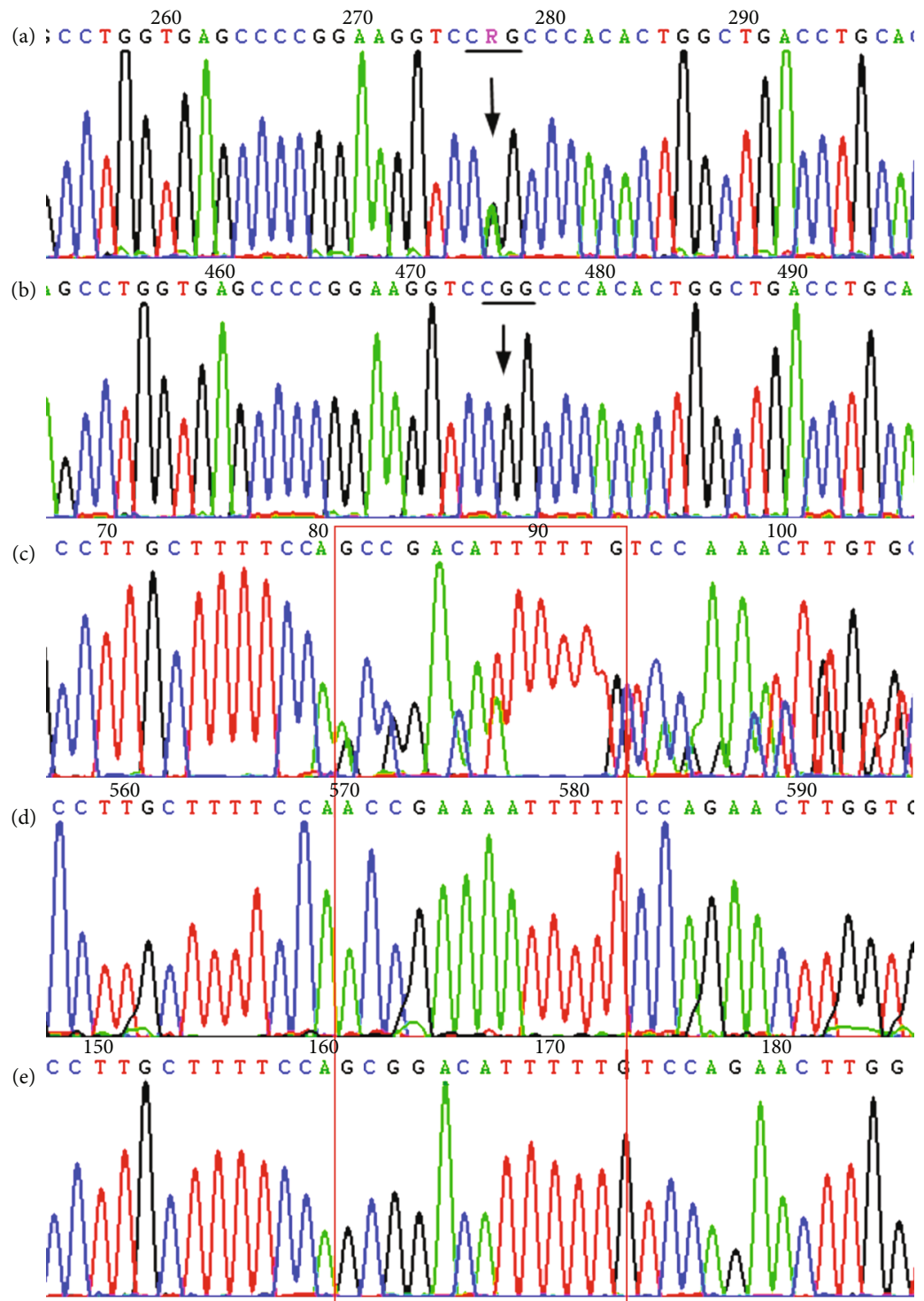

FIGURE 2: Sanger sequencing image of Pedigree A mutation type. (a) The NP_001119580.2:p.Arg83Gln (NM_001126108.2:c.248G>A, rs768527231) heterozygous mutant type in SLC12A3 exon1; (b) the corresponding wild type; (c) the NC_000016.10:g.56872655_56872667 (gcggacatttttg>accgaaaatttt, rs1215667472) heterozygous mutant type in exon8; (d) the clone of the mutant type at the corresponding position; and (e) the clone of the wild type at the corresponding position.

electrocardiogram, even if the electrocardiogram is normal. A dynamic electrocardiogram and exercise electrocardiogram examination should be arranged to identify hidden risks.GS patients are at high risk of chronic kidney disease (CKD); however, this mechanism is complex and has not been clearly elucidated. Chronic hypokalemia can cause renal injury through tubular vacuolization, cysts, and tubulointerstitial nephritis [18], and the pathological report of proband $B$ in this study confirms these results. Some scholars also believe that the increase in circulating renin, angiotensin II, and aldosterone may be more important factors for renal injury and fibrosis [19]. Therefore, it is necessary to closely monitor the renal function indicators of GS patients in clinical practice to improve the prognosis of patients.

It is interesting to note that proband $\mathrm{A}$ and the older sisters of proband B both had thyroid dysfunction. The pro- band A thyroid function test indicated that the patient had autoimmune thyroid disease (AITD) and subclinical hypothyroidism, while the older sister of proband B had AITD and subclinical hyperthyroidism. This information, combined with the color Doppler ultrasound results, and the elevated thyroid peroxidase and TG antibodies, evoked a diagnosis in both of "Hashimoto's thyroiditis (HT)." In the literature review of 18 cases of AITD combined with GS, 13 cases had toxic diffuse goiter (Graves' disease, GD), 3 cases had HT, 2 cases were positive for the simple AITD antibody, and 1 case was a base deletion mutation; the rest were single base substitutions [20]. In the Japanese GS population, the incidence of thyroid dysfunction is $4.3 \%$ [21]. The above studies all indicate that GS is associated with thyroid dysfunction, and the incidence could be higher than expected; however, there is still insufficient data to determine whether 
TABLE 3: Summary on 2 pedigrees with GS family with SCL12A3 gene (Nm_001126108.2) mutant genotype.

\begin{tabular}{|c|c|c|c|c|c|}
\hline & Nucleotide change & Mutation & Predicted effect & Exon & $\begin{array}{l}\text { Number in } \\
\text { dbSNP }\end{array}$ \\
\hline \multicolumn{6}{|l|}{ Pedigree A } \\
\hline & c. $248 \mathrm{G}>\mathrm{A}$ & p.Arg83Gln & Het, missense & 1 & rs768527231 \\
\hline II5,6 & $\begin{array}{c}\text { NC_000016.10:g.56872655_56872667 } \\
\text { (gcggacatttttg>accgaaaatttt) }\end{array}$ & & $\begin{array}{c}\text { Het, splice site/frame } \\
\text { shift }\end{array}$ & 8 & \\
\hline II 4 & C. $248 \mathrm{G}>\mathrm{A}$ & p.Arg83Gln & Het, missense & 1 & rs768527231 \\
\hline $\begin{array}{l}\text { I2; II } 1,3 \text {; } \\
\text { III1, } 2\end{array}$ & $\begin{array}{c}\text { NC_000016.10:g.56872655_56872667 } \\
\text { (gcggacatttttg>accgaaaatttt) }\end{array}$ & & $\begin{array}{c}\text { Het, splice site/frame } \\
\text { shift }\end{array}$ & 8 & \\
\hline \multicolumn{6}{|l|}{ Pedigree B } \\
\hline \multirow{2}{*}{ II 2,3} & c. $2516 \mathrm{~A}>\mathrm{T}$ & p.Asp839Val & Het, missense & 21 & \\
\hline & c. $2711 \mathrm{G}>\mathrm{A}$ & p.Arg904Gln & Het, missense & 23 & rs11643718 \\
\hline I1; III1, 2 & c. $2516 \mathrm{~A}>\mathrm{T}$ & p.Asp839Val & Het, missense & 21 & \\
\hline I2; III3 & c. $2711 \mathrm{G}>\mathrm{A}$ & p.Arg904Gln & Het, missense & 23 & rs11643718 \\
\hline
\end{tabular}

Het: heterozygous mutation.

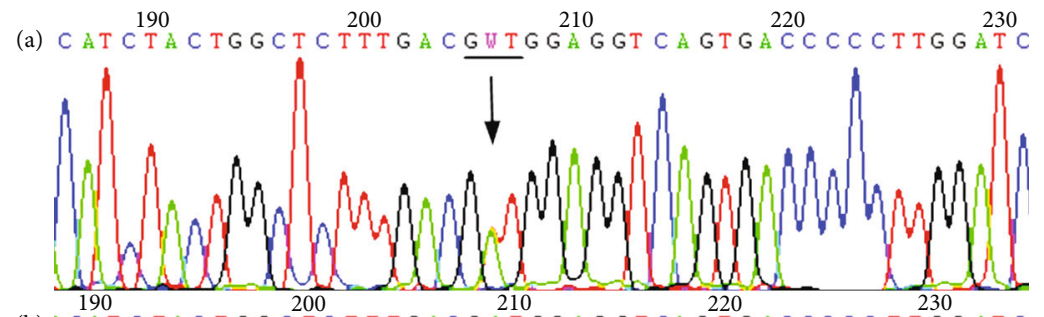

(b) ACATCTACTGGCTCTTTGACGATGGAGGTCAGTGACCCCCTTGGATC

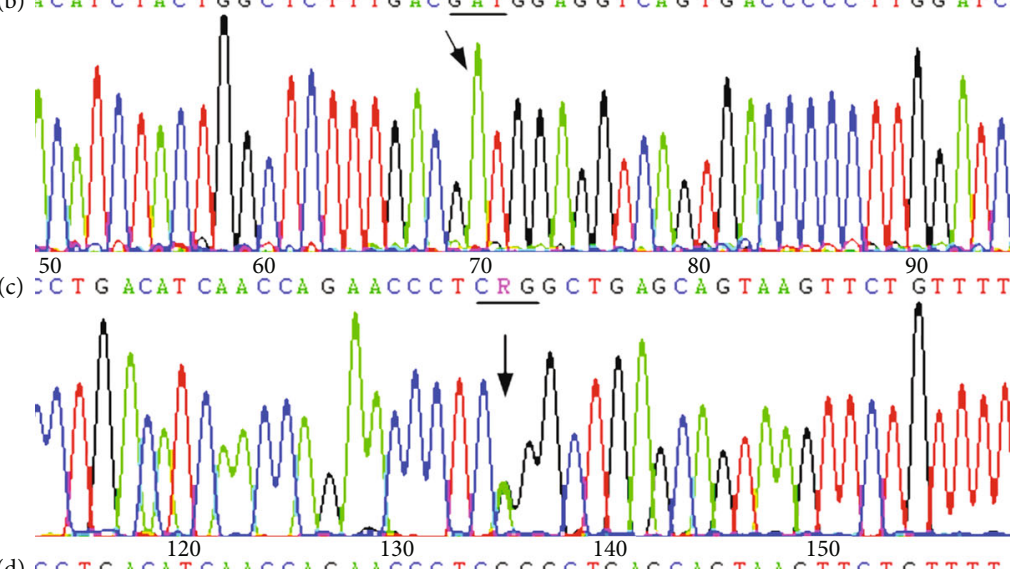

(d) C C T GACATCAACCAGA ACC T T G GGCTGAGCAGTA A GTTCTGTTT T

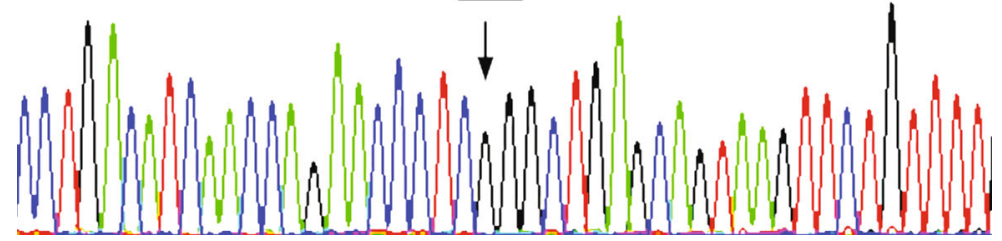

Figure 3: Sanger sequencing diagram of Pedigree B mutation type. (a) The Asp839Val (c.2516A>T) heterozygous mutant type in SLC12A3 exon21; (b) the corresponding wild type; (c) the NP_001119580.2:p.Arg904Gln (NM_001126108.2:c.2711G>A, rs11643718) heterozygous mutant type in exon23; (d) the wild type at the corresponding position. 
AITD is more likely to occur in GS patients than other groups. Although there are sufficient data to suggest that hyperthyroidism can lead to hypokalemia and hypomagnesemia, there is still a lack of research on the long-term effects of these on thyroid function, although iodine metabolism and magnesium metabolism are closely related [22]. Along-term high-magnesium diet leads to thyroid dysfunction, whereas hypomagnesemia may lead to a rapid recurrence of GD, and magnesium supplementation can promote the normalization of thyroid morphology and function [23]. AITD is a complex genetic disease, and the genes leading to AITD disease can be divided into two categories: immunomodulatory genes, including human leukocyte antigen (HLA), cytotoxic T lymphocyte-associated antigen $4(C T L A-4)$, protein tyrosine phosphatases, nonreceptor type 22 (PTPN22), CD40, CD25, and $\mathrm{Fc}$ receptor-like 3 (FCRL3) genes, as well as thyroidspecific genes, including thyroid-stimulating hormone receptor (TSHR) and thyroglobulin $(T g)$; however, currently, there is no evidence that any of these genes are associated with SLC12A3 [20]. Thyroid dysfunction affects kidney physiology and development, and conversely, kidney disease may cause thyroid dysfunction. Hyperthyroidism leads to increased glomerular filtration rates and activation of the reninangiotensin-aldosterone system [24]. Patients with thyroid disease may experience symptoms of GS [25]. Many kidney diseases such as chronic kidney disease and glomerulonephritis are related to thyroid dysfunction, including reduced serum T3, T4, or Hashimoto's thyroiditis [26-28]. Most GS patients are referred to the endocrinology department, as thyroid dysfunction can lead to hypokalemia and hypomagnesemia. For this reason, compared with healthy people, GS patients receive thyroid function tests more frequently.

As of today (24/04/2021), the Clinvar database contains 444 mutations with clinical significance, including conflicting interpretations (23), benign (51), likely benign (73), uncertain significance (150), likely pathogenic (44), and pathogenic (103). The database also contains frameshift, missense, nonsense, splice site, ncRNA, UTR, and other mutation molecular consequences, of which missense is the most common (https:// www.ncbi.nlm.nih.gov/clinvar/?term=SLC12A3). Compound heterozygous mutations are more common than homozygous mutations [6]. Among Chinese GS patients, compound heterozygous mutations accounted for $72.5 \%$, and missense mutations accounted for more than $72 \%$ of different mutations in the $S L C 12 A 3$ gene [29]. The missense mutation Arg83Gln is more common in the GS pedigree, but the pathogenic mechanism remains unclear [30]. Zeng et al. found that among the 137 cases of GS patients in China, six patients carried compound heterozygotes of Arg83Gln, among which two patients also carried three responsible mutation points [29]. The mutant found in proband A: NC_000016.10:g.56872655 56872667 (gcggacattttg $>$ accgaaaatttt) is composed of multiple mutations, including NM_001126108.2:c.976delG mutation, which has been confirmed to be pathogenic [31, 32]. Some researchers believe that the NC_000016.10:g.56872655_ 56872667 (gcggacattttg $>$ accgaaaatttt) mutation is a deletion of the genomic region containing a part of exon 8 (C.965-1 976 delinsacgaaatttt) of SLC12A3, which is expected to destroy RNA splints and possibly lead to the deletion or destruction of protein products. This mutation has been described as "c.965-1 969delGCGGACinsACCGAAA \& c.976 977delGT" and "Intron $7 \quad 1 \mathrm{G}>\mathrm{A} \& \mathrm{Ex} 8 \mathrm{nt}+1$ to +12 delCGGACATTT TTGinsCCGAAAATTTT" [33-35]. Some researchers have suggested that the upregulation of TRPV5/6 and of ROMK1 and Maxi- $\mathrm{K}$ are involved in the pathogenesis of hypocalciuria and hypokalemia in NCC Ser707X knockin mice and human GS, respectively [33]. Glaudemans et al. found that the Thr392Ile mutant did not show transport activity, while the Asn442Ser and Gln1030Arg NCC mutants showed reduced NCC plasma membrane localization and therefore a reduced function of NCC, which may relate to an impaired transport function. The experiment also revealed that the transporters could still reach the plasma membrane even if the $\mathrm{NaCl}$ absorption of NCC mutants Glu121Asp, Pro751Leu, Ser475Cys, and Tyr489His was blocked, indicating that they affect the ion affinity of NCC [34]. From the study of the deletion function of these mutants, we found that the NC_000016.10:g.56872655 56872667 (gcggacatttttg $>$ accgaaaatttt) mutation can be classified as pathogenic. The mother of proband A alone carried the heterozygous mutation NC_000016.10: g.56872655 56872667 (gcggacatttttg>accgaaaatttt), and according to the recessive inheritance rule, the carrier should be clinically and metabolically asymptomatic, but she had long-term hypokalemia. It was reported that about $18-40 \%$ of patients clinically diagnosed with GS carried only one allele mutation in SLCl2A3, detected by direct sequencing [30]. Among the 67 Chinese GS patients, the screening also found that $16.4 \%$ only carried one mutant allele [36]. The possible reasons for this situation are (1) the mutation site may be located in the unsequenced SLCl2A3 regulatory fragment, such as the $5^{\prime}$ or $3^{\prime}$ untranslated region, promoter and enhancer regions, or intron depth [37]; (2) there may be large-scale gene recombination, involving one or more exons, which are difficult to detect by single exon sequencing [38]; (3) the expression of NCCT cotransporters may be affected by epigenetic modifications and/or silent polymorphism, which interfere with its function [38]; and (4) other pathogenic gene mutations may be related to GS. Zelikovic et al. found that, in addition to $S L C 12 A 3$, the R $438 \mathrm{H}$ mutation of $C L C N K B$ may play a role in the pathogenesis of GS [39]. Among the 137 GS patients in China, 9 patients were found to carry the Arg904Gln mutation, and therefore, these can be regarded as frequent mutations [29]. Arg904Gln may be an area for increased research in Chinese GS patients [36]. Bioinformatics analysis showed that if the wild-type 904Arg was replaced by the mutant allele $904 \mathrm{Gln}$, the three-dimensional structure of the SLC12A3 protein will change significantly, and the Arg904Gln mutation may have important physiological significance [40]. Tanaka et al. believed that the Arg904Gln gene variation in SLC12A3 could reduce the risk of diabetic nephropathy in type 2 diabetes mellitus (T2DM) [41]; however, other studies have provided evidence supporting the correlation between Arg904Gln variant and the disease development of diabetic nephropathy in patients with T2DM and GS, suggesting that this variant may be a key predictor of endstage renal disease [42, 43]. Several suspected pathogenic mutations were found near our newly discovered heterozygous mutant of Asp839Val (c.2516A >T): C.2490C $>$ T (p.Thr830=), 
c.2495A > G (p.Asp832Gly), c.2532G>A (p.Trp844Ter), c.2510_ 2511del (p.Leu836_Phe837insTer), c.2514C >T (p.Asp838=), c.2521G $>$ A (p.Gly841Ser), c.2533del (p.Leu845fs), and c.2546T $>$ A (p.Leu849His) (https://www.ncbi.nlm.nih.gov/ clinvar). These mutations can lead to protein product deletion or destruction, and we speculate that since Asp839Val is located in this region, it may also be a pathogenic mutation.

Individualized lifelong oral potassium or magnesium supplementation or both are the main methods for the treatment of patients with GS. In the case of hypomagnesemia, magnesium supplementation is the initial treatment, as magnesium supplementation promotes potassium supplementation and reduces the risk of tetany and other complications [44, 45]. The current consensus recommends that the therapeutic targets of serum potassium and magnesium are $3.0 \mathrm{mmol} / \mathrm{L}$ and $0.6 \mathrm{mmol} / \mathrm{L}$, respectively $[46,47]$. If there is persistent and symptomatic hypokalemia, poor efficacy of the supplements, or an unacceptable level of side effects, other medications can be used, such as potassium-sparing diuretics [48, 49], renin angiotensin system blockers [50], or nonsteroidal anti-inflammatory drugs, such as indomethacin. A combination of both kinds of treatment can also be [51-53] recommended. However, angiotensin-converting enzyme inhibitors (ACEI) and angiotensin receptor blocking (ARB) are not recommended for treating GS due to their increased risk of hypovolemia. The potassium-sparing diuretics amiloride, spironolactone, and eplerenone are all useful for the treatment of GS, as they increase the serum potassium levels in patients resistant to potassium supplementation, and also treat magnesium deficiency worsened by elevated aldosterone levels [48]. Indomethacin is rarely used in GS because plasma prostaglandin E2 (PGE2) levels in GS patients are usually normal. However, some GS patients have significantly higher levels of PGE 2 and PGE 2 metabolites (PGEM) in urine and plasma, while elevated urine PGEM levels indicated severe clinical manifestations, and the COX 2 inhibitor may be a potential therapeutic target in GS patients with increased PGEM [54]. In an open, randomized, crossover study comparing the efficacy and safety of $75 \mathrm{mg}$ indomethacin, $150 \mathrm{mg}$ eplerenone, and $20 \mathrm{mg}$ amiloride in GS patients, each drug increased plasma potassium levels by about $0.3 \mathrm{mmol} / \mathrm{L}$ [55]. Although effective against hypokalemia, indomethacin and other nonsteroidal anti-inflammatory drugs should be used cautiously because of their short- and long-term gastrointestinal side effects and nephrotoxicity. It is worth noting that proton pump inhibitors can affect the biological activity of magnesium, thereby reducing its intestinal absorption [56]. Therefore, it is recommended to increase the intake dose of magnesium appropriately if proband $\mathrm{A}$ is taking proton pump inhibitors in relation to chronic gastritis.

In summary, SLCI $2 A 3$ test not only helps in the diagnosis and treatment of clinically suspected GS patients but can also be used to screen the family members of patients, to provide for the early detection, prevention, and treatment of the disease. In this study, we found relevant new pathogenic mutations and also found that some GS patients with long-term hypokalemia can contract kidney damage, which has been confirmed by kidney tissue biopsies; however, whether GS patients are susceptible to complicated thyroid disease needs to be confirmed by a large sample of evidence-based research. We also summarized the new advances in GS treatment, hoping to help clinical workers better understand the pathogenesis and physiological processes of the disease.

\section{Conclusion}

It was found that SLC12A3 gene detection contributes to the diagnosis of GS, and the newly discovered SLC12A3 mutation enriches the GS gene mutation spectrum. Chronic GS can cause renal impairment. Whether GS patients are susceptible to complicated thyroid disease needs to be confirmed by a large sample of evidence-based medicine.

\section{Data Availability}

The datasets used and/or analyzed during the present study are available from the corresponding author upon reasonable request.

\section{Conflicts of Interest}

The authors do not have any conflicts of interest to declare.

\section{Authors' Contributions}

L.J.W conceived the initial project and conducted sample and data collection. Z.J.H analyzed the data and wrote the manuscript. All authors have read and agreed to the publication of this article. Jian-hui Zhang, Dan-dan Ruan, Ya-nan $\mathrm{Hu}$, and Xing-lin Ruan contributed equally to this work.

\section{Acknowledgments}

We would like to thank Editage (http://www.editage.cn) for English language editing. This work was supported in part by grants from the National Natural Science Foundation of China (No. 81874379), the Fujian Province Medical Innovation Foundation (2019-CXB-3,-4), Fujian Province Natural Science Fund Project (2020J011064), and Fujian Province Joint Funds for the Innovation of Science and Technology (2018Y9011).

\section{References}

[1] H. J. Gitelman, J. B. Graham, and L. G. Welt, "A new familial disorder characterized by hypokalemia and hypomagnesemia," Transactions of the Association of American Physicians, vol. 79, pp. 221-235, 1966.

[2] N. V. Knoers and E. N. Levtchenko, "Gitelman syndrome," Orphanet Journal of Rare Diseases, vol. 3, no. 1, p. 22, 2008.

[3] Y. J. Hsu, S. S. Yang, N. F. Chu, H. K. Sytwu, C. J. Cheng, and S. H. Lin, "Heterozygous mutations of the sodium chloride cotransporter in Chinese children: prevalence and association with blood pressure," Nephrology, Dialysis, Transplantation, vol. 24, no. 4, pp. 1170-1175, 2008.

[4] N. Tago, Y. Kokubo, N. Inamoto, H. Naraba, H. Tomoike, and N. Iwai, "A high prevalence of Gitelman's syndrome mutations in Japanese," Hypertension Research, vol. 27, no. 5, pp. 327331,2004 
[5] M. R. Pollak, V. B. Delaney, R. M. Graham, and S. C. Hebert, "Gitelman's syndrome (Bartter's variant) maps to the thiazide-sensitive cotransporter gene locus on chromosome $16 \mathrm{q} 13$ in a large kindred," Journal of the American Society of Nephrology, vol. 7, no. 10, pp. 2244-2248, 1996.

[6] Q. Lü, Y. Zhang, C. Song et al., "A novel SLC12A3 gene homozygous mutation of Gitelman syndrome in an Asian pedigree and literature review," Journal of Endocrinological Investigation, vol. 39, no. 3, pp. 333-340, 2016.

[7] J. Luo, X. Yang, J. Liang, and W. Li, “A pedigree analysis of two homozygous mutant Gitelman syndrome cases," Endocrine Journal, vol. 62, no. 1, pp. 29-36, 2015.

[8] A. Blanchard, D. Bockenhauer, D. Bolignano et al., "Gitelman syndrome: consensus and guidance from a Kidney Disease: Improving Global Outcomes (KDIGO) Controversies Conference," Kidney International, vol. 91, no. 1, pp. 24-33, 2017.

[9] N. Mastroianni, M. De Fusco, M. Zollo et al., "Molecular cloning, expression pattern, and chromosomal localization of the human Na-Cl thiazide-sensitive cotransporter (SLC12A3)," Genomics, vol. 35, no. 3, pp. 486-493, 1996.

[10] E. Riveira-Munoz, Q. Chang, R. J. Bindels, and O. Devuyst, "Gitelman's syndrome: towards genotype-phenotype correlations?," Pediatric Nephrology, vol. 22, no. 3, pp. 326-332, 2007.

[11] E. Kardalas, S. A. Paschou, P. Anagnostis, G. Muscogiuri, G. Siasos, and A. Vryonidou, "Hypokalemia: a clinical update," Endocrine Connections, vol. 7, no. 4, pp. R135-R146, 2018.

[12] H. Ren, L. Qin, W. Wang et al., "Abnormal glucose metabolism and insulin sensitivity in Chinese patients with Gitelman syndrome," American Journal of Nephrology, vol. 37, no. 2, pp. 152-157, 2013.

[13] O. Melander, M. Orho-Melander, K. Bengtsson et al., "Genetic variants of thiazide-sensitive $\mathrm{NaCl}$-cotransporter in Gitelman's syndrome and primary hypertension," Hypertension, vol. 36, no. 3, pp. 389-394, 2000.

[14] A. Matsuo, T. Katsuya, K. Ishikawa et al., "G2736A polymorphism of thiazide-sensitive $\mathrm{Na}-\mathrm{Cl}$ cotransporter gene predisposes to hypertension in young women," Journal of Hypertension, vol. 22, no. 11, pp. 2123-2127, 2004.

[15] T. Matayoshi, K. Kamide, S. Takiuchi et al., "The thiazidesensitive $\mathrm{Na}(+)-\mathrm{Cl}(-)$ cotransporter gene, $\mathrm{C} 1784 \mathrm{~T}$, and adrenergic receptor-beta3 gene, T727C, may be gene polymorphisms susceptible to the antihypertensive effect of thiazide diuretics," Hypertension Research, vol. 27, no. 11, pp. 821833, 2004.

[16] K. Patel, J. V. McCoy, and P. M. Davis, "Recognizing thyrotoxic hypokalemic periodic paralysis," Journal of the American Academy of Physician Assistants, vol. 31, no. 1, pp. 31-34, 2018.

[17] A. Bettinelli, C. Tosetto, G. Colussi, G. Tommasini, A. Edefonti, and M. G. Bianchetti, "Electrocardiogram with prolonged QT interval in Gitelman disease," Kidney International, vol. 62, no. 2, pp. 580-584, 2002.

[18] M. H. Tseng, S. S. Yang, Y. J. Hsu et al., "Genotype, phenotype, and follow-up in Taiwanese patients with salt-losing tubulopathy associated with SLC12A3 mutation," The Journal of Clinical Endocrinology and Metabolism, vol. 97, no. 8, pp. E1478E1482, 2012.

[19] S. B. Walsh, E. Unwin, R. Vargas-Poussou, P. Houillier, and R. Unwin, "Does hypokalaemia cause nephropathy? An observational study of renal function in patients with Bartter or Gitelman syndrome," QJM, vol. 104, no. 11, pp. 939-944, 2011.
[20] H. Zhou, X. Liang, Y. Qing et al., "Complicated Gitelman syndrome and autoimmune thyroid disease: a case report with a new homozygous mutation in the SLC12A3 gene and literature review," BMC Endocrine Disorders, vol. 18, no. 1, p. 82, 2018.

[21] A. Al-Shibli, M. Yusuf, I. Abounajab, and P. J. Willems, "Mixed Bartter-Gitelman syndrome: an inbred family with a heterogeneous phenotype expression of a novel variant in the CLCNKB gene," Springerplus, vol. 3, no. 1, p. 96, 2014.

[22] A. K. Chandra, H. Goswami, and P. Sengupta, "Effects of magnesium on cytomorphology and enzyme activities in thyroid of rats," Indian Journal of Experimental Biology, vol. 52, no. 8, pp. 787-792, 2014.

[23] R. Moncayo and H. Moncayo, "The WOMED model of benign thyroid disease: acquired magnesium deficiency due to physical and psychological stressors relates to dysfunction of oxidative phosphorylation," BBA Clinical, vol. 3, pp. 44-64, 2015.

[24] J. G. den Hollander, R. W. Wulkan, M. J. Mantel, and A. Berghout, "Correlation between severity of thyroid dysfunction and renal function," Clinical Endocrinology, vol. 62, no. 4, pp. 423-427, 2005.

[25] N. Aoi, T. Nakayama, Y. Tahira et al., "Two novel genotypes of the thiazide-sensitive $\mathrm{Na}-\mathrm{Cl}$ cotransporter (SLC12A3) gene in patients with Gitelman's syndrome," Endocrine, vol. 31, no. 2, pp. 149-153, 2007.

[26] B. Akikusa, Y. Kondo, Y. Iemoto, K. Iesato, and M. Wakashin, "Hashimoto's thyroiditis and membranous nephropathy developed in progressive systemic sclerosis (PSS)," American Journal of Clinical Pathology, vol. 81, no. 2, pp. 260-263, 1984.

[27] M. R. Wiederkehr, J. Kalogiros, and R. Krapf, "Correction of metabolic acidosis improves thyroid and growth hormone axes in haemodialysis patients," Nephrology, Dialysis, Transplantation, vol. 19, no. 5, pp. 1190-1197, 2004.

[28] G. Basu and A. Mohapatra, "Interactions between thyroid disorders and kidney disease," Indian Journal of Endocrinology and Metabolism, vol. 16, no. 2, pp. 204-213, 2012.

[29] Y. Zeng, P. Li, S. Fang et al., "Genetic analysis of SLC12A3 gene in Chinese patients with Gitelman syndrome," Medical Science Monitor, vol. 25, pp. 5942-5952, 2019.

[30] R. Vargas-Poussou, K. Dahan, D. Kahila et al., "Spectrum of mutations in Gitelman syndrome," Journal of the American Society of Nephrology, vol. 22, no. 4, pp. 693-703, 2011.

[31] R. R. Shi, C. C. Li, L. Fang et al., "Clinical and genetic characteristics of Gitelman syndrome in 5 pedigrees," Zhonghua Nei Ke Za Zhi, vol. 56, no. 2, pp. 104-111, 2017.

[32] L. Shao, L. Liu, Z. Miao et al., "A novel SLC12A3 splicing mutation skipping of two exons and preliminary screening for alternative splice variants in human kidney," American Journal of Nephrology, vol. 28, no. 6, pp. 900-907, 2008.

[33] S. S. Yang, Y. F. Lo, I. S. Yu et al., "Generation and analysis of the thiazide-sensitive $\mathrm{Na}+-\mathrm{Cl}$ - cotransporter (Ncc/Slc12a3) Ser707X knockin mouse as a model of Gitelman syndrome," Human Mutation, vol. 31, no. 12, pp. 1304-1315, 2010.

[34] B. Glaudemans, H. G. Yntema, P. San-Cristobal et al., "Novel NCC mutants and functional analysis in a new cohort of patients with Gitelman syndrome," European Journal of Human Genetics, vol. 20, no. 3, pp. 263-270, 2012.

[35] L. Wang, C. Dong, Y. G. Xi, and X. Su, "Thiazide-sensitive $\mathrm{Na}+\mathrm{Cl}$ - cotransporter: genetic polymorphisms and human diseases," Acta Biochimica et Biophysica Sinica Shanghai, vol. 47, no. 5, pp. 325-334, 2015. 
[36] B. Zha, P. Zheng, J. Liu, and X. Huang, "Coexistence of Graves' Disease in a 14-year-old young girl with Gitelman Syndrome," Clinical Endocrinology, vol. 83, no. 6, pp. 995-997, 2015.

[37] S. H. Lin, J. C. Shiang, C. C. Huang, S. S. Yang, Y. J. Hsu, and C. J. Cheng, "Phenotype and genotype analysis in Chinese patients with Gitelman's syndrome," The Journal of Clinical Endocrinology and Metabolism, vol. 90, no. 5, pp. 2500-2507, 2005.

[38] F. Nakhoul, N. Nakhoul, E. Dorman, L. Berger, K. Skorecki, and D. Magen, "Gitelman's syndrome: a pathophysiological and clinical update," Endocrine, vol. 41, no. 1, pp. 53-57, 2012.

[39] I. Zelikovic, R. Szargel, A. Hawash et al., "A novel mutation in the chloride channel gene,_CLCNKB_, as a cause of Gitelman and Bartter syndromes," Kidney International, vol. 63, no. 1, pp. 24-32, 2003.

[40] N. Abu Seman, B. He, J. R. Ojala et al., "Genetic and biological effects of sodium-chloride cotransporter (SLC12A3) in diabetic nephropathy," American Journal of Nephrology, vol. 40, no. 5, pp. 408-416, 2014.

[41] N. Tanaka, T. Babazono, S. Saito et al., "Association of solute carrier family 12 (sodium/chloride) member 3 with diabetic nephropathy, identified by genome-wide analyses of single nucleotide polymorphisms," Diabetes, vol. 52, no. 11, pp. 2848-2853, 2003.

[42] R. Zhang, L. Zhuang, M. Li et al., "Arg913Gln of SLC12A3 gene promotes development and progression of end-stage renal disease in Chinese type 2 diabetes mellitus," Molecular and Cellular Biochemistry, vol. 437, no. 1-2, pp. 203-210, 2018.

[43] E. de la Cruz-Cano, C. . C. Jiménez-González, V. Morales-García et al., "Arg913Gln variation of SLC12A3 gene is associated with diabetic nephropathy in type 2 diabetes and Gitelman syndrome: a systematic review," BMC Nephrology, vol. 20, no. 1, p. 393, 2019.

[44] Z. S. Agus, "Hypomagnesemia," Journal of the American Society of Nephrology, vol. 10, no. 7, pp. 1616-1622, 1999.

[45] N. V. Knoers, "Gitelman syndrome," Advances in Chronic Kidney Disease, vol. 13, no. 2, pp. 148-154, 2006.

[46] G Gitelman Syndrome Collaborative Study, "Expert consensus for the diagnosis and treatment of patients with Gitelman syndrome," Zhonghua Nei Ke Za Zhi, vol. 56, no. 9, pp. 712-716, 2017.

[47] H. Gallagher, J. Soar, and C. Tomson, "New guideline for perioperative management of people with inherited salt- wasting alkaloses," British Journal of Anaesthesia, vol. 116, no. 6, pp. 746-749, 2016.

[48] G. Colussi, G. Rombol\&agrave, M. E. de Ferrari, M. Macaluso, and L. Minetti, "Correction of hypokalemia with antialdosterone therapy in Gitelman's syndrome," American Journal of Nephrology, vol. 14, no. 2, pp. 127-135, 1994.

[49] Y. Ito, M. Yoshida, M. Nakayama et al., "Eplerenone improved hypokalemia in a patient with Gitelman's syndrome," Internal Medicine, vol. 51, no. 1, pp. 83-86, 2012.

[50] G. Brambilla, M. Perotti, S. Perra, R. Dell'Oro, G. Grassi, and A. I. Pincelli, "It is never too late for a genetic disease: a case of a 79-year-old man with persistent hypokalemia," Journal of Nephrology, vol. 26, no. 3, pp. 594-598, 2013.

[51] N. Larkins, M. Wallis, B. McGillivray, and C. Mammen, "A severe phenotype of Gitelman syndrome with increased prostaglandin excretion and favorable response to indomethacin," Clinical Kidney Journal, vol. 7, no. 3, pp. 306-310, 2014.
[52] L. C. Liaw, K. Banerjee, and M. G. Coulthard, "Dose related growth response to indometacin in Gitelman syndrome," Archives of Disease in Childhood, vol. 81, no. 6, pp. 508-510, 1999.

[53] H. Mayan, O. Gurevitz, and Z. Farfel, "Successful treatment by cyclooxyenase- 2 inhibitor of refractory hypokalemia in a patient with Gitelman's syndrome," Clinical Nephrology, vol. 58, no. 7, pp. 73-76, 2002.

[54] X. Peng, L. Jiang, C. Chen et al., "Increased urinary prostaglandin E2 metabolite: a potential therapeutic target of Gitelman syndrome," PLoS One, vol. 12, no. 7, article e0180811, 2017.

[55] A. Blanchard, R. Vargas-Poussou, M. Vallet et al., "Indomethacin, amiloride, or eplerenone for treating hypokalemia in Gitelman syndrome," Journal of the American Society of Nephrology, vol. 26, no. 2, pp. 468-475, 2015.

[56] G. Famularo, L. Gasbarrone, and G. Minisola, "Hypomagnesemia and proton-pump inhibitors," Expert Opinion on Drug Safety, vol. 12, no. 5, pp. 709-716, 2013. 